\title{
26239 - IMPLICATIONS OF PARAVALVULAR LEAKS AFTER VALVE REPLACEMENT SURGERY
}

\author{
Jacek Karski MD, Wei Xu, MD; Ludwik Fedorko, MD; Yuji Hirisaki; George \\ Djaiani, MD \\ Toronto General Hospital, UHN, Toronto, ONTARIO, Canada
}

INTRODUCTION: Perivalvular leaks (PVL) after aortic (AVR) and mitral valve replacement (MVR) surgery may significantly contribute to patients' short and long-term morbidity. A recent report has identified that $6 \%$ and $32 \%$ of patients undergoing AVR and MVR respectively had PVL in the operating room.(1) We reviewed the rates of intraoperative PVL's as detected by intraoperative transesophageal echocardiography (TEE) and perioperative morbidity of these patients.

METHODS: After Research Ethics Board approval, we reviewed demographic data, surgical characteristics and TEE reports on 442 patients scheduled for AVR and/or MVR surgery between December 2002 and January 2004. PVL was classed as trivial, mild, moderate or severe. All patients were divided into two groups: with and without PVL. Groups were compared using ANOVA and Chi-square analysis. Multivariant analysis was performed to identify risk factors for PVL.

RESULTS: A total of 52(12\%) patients had PVL intraoperatively, 23 after AVR (7 mild, 3 moderate, and 13 severe), and 29 after MVR (13 trivial, 7 mild, 4 moderate, and 5 severe). Transthoracic echocardiography (TTE) was performed 1-week after surgery, and TTE was done 1-year after surgery. All severe PVL's were repaired intraoperatively after AVR and 4 out of 5 after MVR. One patient with severe PVL post MVR required reoperation and died postoperatively. Only 1 trivial and 1 mild PVL after AVR and 1 mild PVL after MVR were repaired intraoperatively. During TTE examination 1-week after surgery, PVL remained unchanged in 9 patients, regressed in 6-patients, and could not be detected in 31 -patients. In four patients with PVL early TTE, demonstrated increase of severity of PVL and one patient had to be re operated 8 days after primary MVR surgery. One year follow-up TTE demonstrated that all aortic PVL healed. Only in 2 patients with primary PVL after MVR paravalvular leak was demonstrated (one trace and one mild).

Compared to patients without PVL, patients with PVL were considerably older, mean age 67 vs. 63 years, $\mathrm{p}=0.04$, had more commonly congestive heart failure preoperatively, $62 \%$ vs. $47 \%, \mathrm{p}=0.04$, and had higher rate of preoperative renal dysfunction $34 \%$ vs $21 \%$, $\mathrm{p}=0.04$, and longer cardiopulmonary bypass time, mean $146 \mathrm{~min}$ vs. $122 \mathrm{~min}, \mathrm{p}=0.003$. Bioprosthetic MVR was associated with higher prevalence of PVL when compared to mechanical MVR, $63 \%$ vs. $36 \%$, $\mathrm{p}=0.01$.

Patients with postoperative diagnosis of PVL suffered more postoperative complications with prolonged ICU and hospital length of stay. (Table I) DISCUSSION: Prevalence of PVL after MVR and AVR is lower then previously reported. Patients who suffer PVL are older, undergo more complex surgery, and have increased perioperative morbidity, which is associated with longer intensive care unit and hospital length of stay. Only patients with moderate and severe PVL's require immediate repair during primary surgery.

REFERENCE: Am Heart J. 138(2 Pt 1):351-7, 1999 Aug. 
Table I. Patients with perivalvular leak (PVL) vs. patients without PVL.

Data expressed as mean $\pm \mathrm{SD}$, or median [range].

\begin{tabular}{|l|c|c|c|}
\hline Variable & PVL present (n=52) & No PVL $(\mathrm{n}=390)$ & P value \\
\hline Preoperative & & & \\
\hline Age (yrs) & $67 \pm 12$ & $63 \pm 13$ & 0.04 \\
\hline Renal Dysfinction (\%) & 33 & 21 & 0.04 \\
\hline History of Heart Failure (\%) & 62 & 46 & 0.04 \\
\hline Intra-operative & & & \\
\hline Mitral bioprosthetic (\%) & 63 & 36 & 0.01 \\
\hline CPB time (minutes) & $149 \pm 57$ & $122 \pm 38$ & 0.003 \\
\hline Postoperative & & & 0.02 \\
\hline ICU LOS (days) & $2[1,5]$ & $2[1,4]$ & 0.002 \\
\hline Hospital LOS (days) & $10[8,12]$ & $8[6,11]$ & 0.02 \\
\hline LCOS (CI < 2lm2) (\%) & 13.2 & 4.4 & 0.04 \\
\hline Atrial Fibrillation (\%) & 41.5 & 27.3 & 0.02 \\
\hline Sepsis (\%) & 9.4 & 2.3 & 0.3 \\
\hline Mortality (\%) & 3.8 & 1.8 & \\
\hline
\end{tabular}

ICU LOS- Intensive Care Unit length of stay, MI" myocardial infarction, LCOS-Low Cardiac

Oupper Syndrome, CPB" casdiopulmonary bypass 